\title{
Characteristic expression of fukutin in gastric cancer among atomic bomb survivors
}

\author{
TRANG T.B. PHAM ${ }^{1}$, NAOHIDE OUE ${ }^{1}$, MANABU YAMAMOTO $^{2}$, MEGUMU FUJHARA $^{3}$, \\ TERUYOSHI ISHIDA ${ }^{4}$, SHOICHIRO MUKAI ${ }^{1}$, NAOYA SAKAMOTO ${ }^{1}$, KAZUHIRO SENTANI ${ }^{1}$ and WATARU YASUI $^{1}$ \\ ${ }^{1}$ Department of Molecular Pathology, Hiroshima University Institute of Biomedical and Health Sciences, Hiroshima 734-8551; \\ ${ }^{2}$ Department of Gastroenterological Surgery, National Kyushu Cancer Center, Fukuoka 811-1395; Departments of ${ }^{3}$ Pathology \\ and ${ }^{4}$ Surgery, Hiroshima Red Cross Hospital and Atomic-Bomb Survivors Hospital, Hiroshima 730-8619, Japan
}

Received February 2, 2016; Accepted October 25, 2016

DOI: $10.3892 / \mathrm{ol} .2016 .5520$

\begin{abstract}
Approximately 70 years have passed since the atomic bombs were dropped on Nagasaki and Hiroshima. To elucidate potential biomarkers and possible mechanisms of radiation-induced cancer, the expression of FKTN, which encodes fukutin protein and causes Fukuyama-type congenital muscular dystrophy, was analyzed in gastric cancer (GC) tissue samples from atomic bomb survivors. Expression of cluster of differentiation (CD) 10 was also evaluated, as it has previously been observed that positive fukutin expression was frequently noted in CD10-positive GC cases. In the first cohort from Hiroshima Red Cross Hospital and Atomic-Bomb Survivors Hospital (Hiroshima, Japan; n=92), 102 (53\%) of the GC cases were positive for fukutin. Expression of fukutin was not associated with exposure status, but was associated with CD10 expression $(\mathrm{P}=0.0001)$. The second cohort was from Hiroshima University Hospital (Hiroshima, Japan; n=86), and these patients were also in the Life Span Study cohort, in which atomic bomb radiation doses were precisely estimated using the DS02 system. Expression of fukutin was detected in $58(67 \%)$ of GC cases. GC cases positive for fukutin were observed more frequently in the low dose-exposed group than in the high dose-exposed group $(\mathrm{P}=0.0001)$. Further studies with a larger cohort, including precise radiation dose estimation, may aid in clarifying whether fukutin could serve as a potential biomarker to define radiation-induced GC in atomic-bomb survivors.
\end{abstract}

Correspondence to: Dr Naohide Oue, Department of Molecular Pathology, Hiroshima University Institute of Biomedical and Health Sciences, 1-2-3 Kasumi, Minami-ku, Hiroshima 734-8551, Japan E-mail: naoue@hiroshima-u.ac.jp

Key words: gastric cancer, radiation carcinogenesis, atomic bomb, fukutin, FKTN

\section{Introduction}

Atomic bombs were dropped on Nagasaki and Hiroshima in August 1945. The Life Span Study (LSS) cohort from the Radiation Effects Research Foundation (RERF) was established and included 120,000 subjects who were exposed to radiation from the atomic bombs (1). During the initial 6-8 years following the bombings, the frequency of leukemia reached a peak and then reduced dramatically to an excess relative risk (ERR) of 0 (2). Conversely, frequencies of various types of solid cancer, including bladder, breast, lung, stomach and brain, were relatively high with an ERR of $0.47(3,4)$.

Gastric cancer (GC) is the fifth most common malignancy in the world and the second highest cause of mortality for men and women (5). Over $60 \%$ of GC cases worldwide occur in Asian countries, including Japan, China, Korea and Vietnam $(6,7)$. While radiation is currently widely used in medicine, industry and nuclear power, the effect of radiation on GC development has been estimated on the basis of LSS with ERRs per Gy of 1.20 for mortality (8) and 1.32 for incidence (1). However, Preston et al (9) estimated that, following exposure at 30 years of age, the risk of cancer at age 70 increased by $35 \%$ per Gy for males and 58\% per Gy for females. Various studies have identified mutations in TP53 and $B R A F$ in atomic bomb survivors (10-12), but no study has examined the specific alterations in radiation-associated cancer. A previous study demonstrated that alteration of gene expression occurs in radiation-associated GC (13). It was observed that versican and osteonectin genes are expressed at much lower levels in tumor-associated stroma in a group of subjects exposed to a high dose of radiation, compared with a group exposed to a low dose (13). In addition, more frequent GC cases with high microRNA (miR)-24 expression levels were noted in the high dose-exposed group than in the low dose-exposed group (14). However, these findings cannot fully explain the pathogenesis of radiation-associated GC.

An Escherichia coli ampicillin secretion trap was performed in previous studies using GC cell lines and tissue samples (15-17), which demonstrated that the FKTN gene is overexpressed in GC (18). FKTN, which encodes fukutin protein, is responsible for Fukuyama-type congenital muscular dystrophy (19). Fukutin is presumed to be involved in the 
glycosylation of $\alpha$-dystroglycan, which functions in basement membrane formation (19). Although overexpression of fukutin has been reported in GC (18), its association with radiation exposure has not yet been investigated.

In the present study, immunohistochemical analysis of fukutin was performed in order to elucidate the association between fukutin expression and radiation-associated GC. In addition, a focus was placed upon the gastric and intestinal mucin phenotype of GC, as fukutin expression is observed more commonly in the intestinal phenotype of GC than in other phenotypes (18).

\section{Materials and methods}

Tissue samples. The present study included formalin-fixed and paraffin-embedded archival tissues from 278 patients with GC who underwent surgery. The patients were treated at Hiroshima Red Cross Hospital and Atomic-Bomb Survivors Hospital (HRCHABSH; Hiroshima, Japan), between April 1991 and March 2000 or Hiroshima University Hospital (HUH; Hiroshima, Japan) between April 1991 and March 2000.

The HRCHABSH cohort included 192 GC samples, all from atomic bomb survivors in Hiroshima treated at HRCHABSH. As these patients were not LSS cohort members, the atomic bomb radiation doses were not estimated. The HRCHABSH cohort patients included directly exposed patients and those not present in Hiroshima city at the time of bombing, but who entered the city soon following the bombing (within 2 weeks). These patients were classified into two groups: The exposed at a short distance group (directly exposed patients, exposure distance from the hypocenter of $\leq 4 \mathrm{~km}$ ) and the exposed at a long distance group (patients not present in Hiroshima city at the time of bombing, but who entered the region $\leq 4 \mathrm{~km}$ from the hypocenter within 2 weeks of the explosion).

The HUH cohort included $86 \mathrm{GC}$ samples, all from atomic bomb survivors in Hiroshima treated at HUH. The HUH cohort of 86 patients with GC were also LSS cohort members, and therefore atomic bomb radiation doses were estimated using the DS02 system (20). These patients were classified into two groups according to the levels of exposed radiation dose: The high dose-exposed group ( $\geq 5 \mathrm{mGy}$ ) and the low dose-exposed group ( $<5 \mathrm{mGy}$ ).

Tumor staging was performed according to the tumor-node-metastasis classification system (21). Histologic classification of GC was performed according to the Lauren classification system (22). The study was approved by The Ethics Committee for Human Genome Research of Hiroshima University (Hiroshima, Japan).

Immunohistochemistry. One or two representative tumor blocks, including the tumor center, invading front and tumor-associated non-neoplastic mucosa, from each patient were examined by immunohistochemistry. In cases of large, late-stage tumors, two varied sections were examined to include representative areas of the tumor center, in addition to the lateral and deep tumor invasive front. Immunohistochemical analysis was performed using the Dako EnVision+ Mouse Peroxidase Detection system (Dako, Glostrup, Denmark). Antigen retrieval was performed by microwave heating in citrate buffer ( $\mathrm{pH}$ 6.0) for $30 \mathrm{~min}$. Peroxidase activity was blocked with $3 \% \mathrm{H}_{2} \mathrm{O}_{2}$-methanol for $10 \mathrm{~min}$ at room temperature, and sections were incubated with normal goat serum (Dako) for $20 \mathrm{~min}$ at room temperature to block nonspecific antibody binding sites. Sections were incubated with a mouse monoclonal anti-fukutin antibody (\#ab131280; dilution, 1:50; Abcam, Cambridge, UK) for $1 \mathrm{~h}$ at room temperature, followed by incubation with Envision+ anti-rabbit peroxidase for $1 \mathrm{~h}$ at room temperature. For the color reaction, sections were incubated with Liquid DAB+ Substrate Chromogen Solution (Dako) for $10 \mathrm{~min}$. Sections were counterstained with $0.1 \%$ hematoxylin, and negative controls were created by omission of the primary antibody.

Expression of fukutin was scored in all tumors as positive or negative. Immunostaining for fukutin was considered positive when $>10 \%$ of tumor cells were stained. Using these definitions, two surgical pathologists (Hiroshima University), without knowledge of the clinical and pathological parameters or the patients' outcomes, independently reviewed immunoreactivity in each specimen. Interobserver differences were resolved by consensus review at a double-headed microscope following independent review.

Phenotypic analysis of GC. GCs were classified into four phenotypes: Gastric (G) type, intestinal (I) type, gastric and intestinal mixed (GI) type and unclassified (N) type. For phenotypic expression analysis of GC, immunohistochemical analysis was performed (as described above) with four antibodies: Anti-Mucin (MUC) 5AC (\#NCL-MUC-5AC; dilution, 1:50; Novocastra; Leica Biosystems, Nussloch, Germany) as a marker of gastric foveolar epithelial cells, anti-MUC6 (\#NCL-MUC-6; dilution, 1:50; Novocastra; Leica Biosystems) as a marker of pyloric gland cells, anti-MUC2 (\#PA0155; dilution, 1:50; Novocastra; Leica Biosystems) as a marker of goblet cells in the small intestine and colorectum and anti-CD10 (\#PA0270; dilution, 1:50; Novocastra; Leica Biosystems) as a marker of microvilli of absorptive cells in the small intestine and colorectum. The criteria for the classification of $\mathrm{G}$ type and I type GCs were as follows (17): GCs in which $>10 \%$ of cells in the section expressed at least one gastric epithelial cell marker (MUC5AC or MUC6) or intestinal epithelial cell marker (MUC2 or CD10) were classified as G type or I type cancers, respectively; sections that exhibited gastric and intestinal phenotypes were classified as GI type; and those that lacked the gastric and intestinal phenotypes were classified as $\mathrm{N}$ type.

Statistical analysis. SPSS version 23.0 was used for all statistical analyses (IBM SPSS, Armonk, NY, USA). Associations between clinicopathological parameters and fukutin protein expression were analyzed using Fisher's exact test. $\mathrm{P}<0.05$ was considered to indicate a statistically significant difference.

\section{Results}

Expression of fukutin in the HRCHABSH cohort. Expression of fukutin was first analyzed in the HRCHABSH cohort. All 192 patients were atomic bomb survivors in Hiroshima, who developed GC following the bombing. This cohort was subdivided into two groups, which comprised of 117 patients in the 
Table I. Clinicopathological characteristics of patients and radiation exposure status in the HRCHABSH cohort.

\begin{tabular}{|c|c|c|c|}
\hline \multirow[b]{2}{*}{ Characteristic } & \multicolumn{2}{|c|}{ Fukutin expression } & \multirow[b]{2}{*}{ P-value } \\
\hline & $\begin{array}{l}\text { Positive, } \\
\text { n (\%) }\end{array}$ & $\begin{array}{c}\text { Negative, } \\
\text { n (\%) }\end{array}$ & \\
\hline Age, years & & & 0.0527 \\
\hline$\leq 65$ & $20(41)$ & $29(59)$ & \\
\hline$>65$ & $82(57)$ & $61(43)$ & \\
\hline Gender & & & 0.9405 \\
\hline Male & $64(53)$ & $56(47)$ & \\
\hline Female & $38(53)$ & $34(47)$ & \\
\hline CD10 expression & & & 0.0001 \\
\hline Positive & $30(83)$ & $6(17)$ & \\
\hline Negative & $72(46)$ & $84(54)$ & \\
\hline Exposure status & & & 0.8025 \\
\hline $\begin{array}{l}\text { Exposed in short } \\
\text { distance group }\end{array}$ & $63(54)$ & $54(46)$ & \\
\hline $\begin{array}{l}\text { Exposed in long } \\
\text { distance group }\end{array}$ & $39(52)$ & $36(48)$ & \\
\hline Stage & & & 0.4470 \\
\hline Stage I/II & $71(55)$ & $58(45)$ & \\
\hline Stage III/IV & $31(49)$ & $32(51)$ & \\
\hline Lauren classification & & & 0.0009 \\
\hline Intestinal & $68(76)$ & $21(24)$ & \\
\hline Diffuse & $34(33)$ & $69(67)$ & \\
\hline
\end{tabular}

HRCHABSH, Hiroshima red cross hospital and atomic-bomb survivors hospital; $\mathrm{CD}$, cluster of differentiation.

group exposed at a short distance and 75 patients in the group exposed at a long distance. Patient characteristics, including age at diagnosis, gender, tumor stage and Lauren classification, did not statistically differ between the two groups (data not shown). As reported previously (18), in non-neoplastic gastric mucosa, immunohistochemical analysis exhibited weak staining of fukutin in intestinal metaplastic cells, but not in the normal gastric epithelial glands. By contrast, GC tissue exhibited stronger, more extensive staining of fukutin. Cytoplasmic granular staining of fukutin was observed in GC cells, from the superficial layer to the deep layer, particularly in intestinal type GC (Fig. 1A). Certain cells of diffuse type GC also demonstrated fukutin staining (Fig. 1B). It has been previously noted that positive fukutin expression is frequently observed in CD10-positive GC cases (18). Therefore, the association between fukutin expression and CD10 expression was subsequently analyzed, and confirmed that positive fukutin expression was frequently observed in CD10-positive GC cases (Fig. 1C). Numerous GC cases demonstrated heterogeneous fukutin staining and the percentage of fukutin-stained GC cells ranged from $0-80 \%$. Staining of $>10 \%$ of tumor cells was considered to be positive for fukutin. In total, 102 (53\%) of $192 \mathrm{GC}$ cases were positive for fukutin.
Table II. Clinicopathological characteristics of patients and radiation exposure status in the HUH cohort.

\begin{tabular}{lcrr}
\hline & \multicolumn{2}{c}{ Fukutin expression } & \\
\cline { 2 - 3 } Characteristic & Positive, n (\%) & Negative, n (\%) & P-value \\
\hline Age, years & & & 0.4297 \\
$\leq 65$ & $13(59)$ & $9(41)$ & \\
$>65$ & $45(70)$ & $19(30)$ & \\
Gender & & & 0.8202 \\
Male & $29(69)$ & $13(31)$ & \\
Female & $29(66)$ & $15(34)$ & \\
CD10 expression & & & 0.7911 \\
$\quad$ Positive & $15(71)$ & $6(29)$ & \\
$\quad$ Negative & $43(66)$ & $22(34)$ & \\
Exposure status & & & 0.0001 \\
High-dose group & $21(48)$ & $23(52)$ & \\
Low-dose group & $37(88)$ & $5(12)$ & \\
Stage & & & 0.2307 \\
$\quad$ Stage I/II & $40(73)$ & $15(27)$ & \\
$\quad$ Stage III/IV & $18(58)$ & $13(42)$ & \\
Lauren classification & & & 0.0160 \\
Intestinal & $43(77)$ & $13(23)$ & \\
Diffuse & $15(50)$ & $15(50)$ & \\
\hline
\end{tabular}

HUH, hiroshima university hospital; CD, cluster of differentiation.

Following this, the associations between fukutin expression and clinicopathological characteristics were examined (Table I). Positive fukutin expression was observed more frequently in intestinal type GC cases than in diffuse type $\mathrm{GC}$ cases $(\mathrm{P}=0.0009$, Fisher's exact test; Table I). In addition, positive fukutin expression was frequently observed in CD10-positive GC cases ( $\mathrm{P}=0.0001$; Table I). By contrast, fukutin expression was not associated with exposure status (Table I) or with gastric/intestinal mucin phenotype (data not shown).

Expression of fukutin in the HUH cohort. In the HRCHABSH cohort, it was demonstrated that fukutin expression was associated with Lauren classification and CD10 expression. However, there was no association between fukutin expression and exposure status. Therefore, analysis of fukutin expression levels in the HUH cohort was performed, as these patients were LSS cohort members, in which atomic bomb radiation doses were estimated correctly by the DS02 system (20). All 86 patients were atomic bomb survivors in Hiroshima and were comprised of 44 high dose-exposed and 42 low dose-exposed patients, who developed GC following the bombing. Patient characteristics, including age at diagnosis, sex, tumor stage and Lauren classification, did not statistically differ between the high dose-exposed and the low dose-exposed groups (data not shown). As with the HRCHABSH cohort, cytoplasmic granular staining of fukutin was observed in the GC cells, from the superficial layer to the deep layer, particularly in 


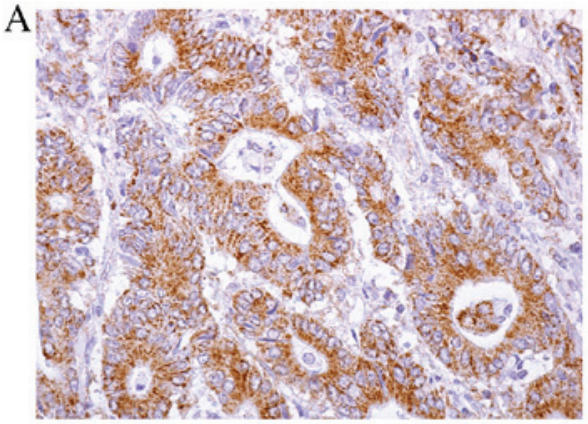

$\mathrm{C}$

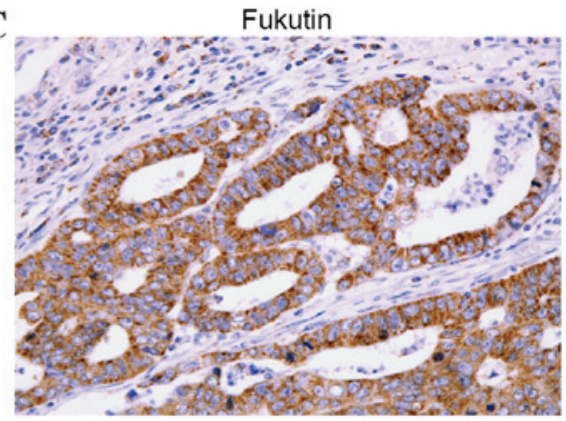

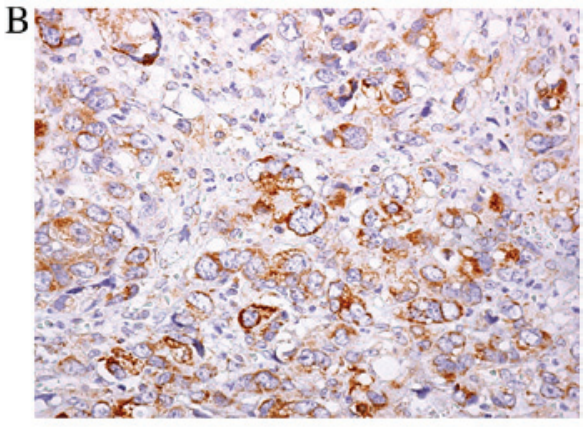

CD10

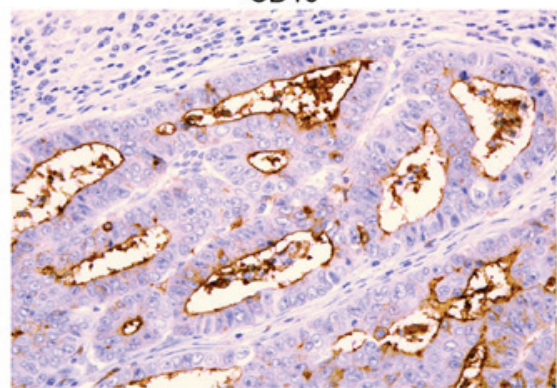

Figure 1. Immunohistochemical analysis of fukutin. (A) Immunohistochemical analysis of fukutin in intestinal type GC (original magnification, x400). (B) Immunohistochemical analysis of fukutin in diffuse type gastric cancer (original magnification, $\mathrm{x} 400$ ). (C) Immunohistochemical analysis of fukutin and CD10 in intestinal type gastric cancer. Gastric cancer cells positive for fukutin were also stained by CD10 (original magnification, x200). GC, gastric cancer; $\mathrm{CD}$, cluster of differentiation.

intestinal type GC (data not shown). A number of GC cases exhibited heterogeneous fukutin staining and the percentage of fukutin-stained GC cells ranged from $0-80 \%$. A total of 58 (67\%) of $86 \mathrm{GC}$ cases were positive for fukutin.

As with the HRCHABSH cohort, positive fukutin expression was observed more frequently in intestinal type GC cases than in diffuse type GC cases $(\mathrm{P}=0.0160$; Table II). In this cohort, fukutin expression was associated with exposure status $(\mathrm{P}=0.0001)$, but not with CD10 expression (Table II). No association was noted between fukutin expression levels and mucin phenotype (data not shown).

\section{Discussion}

As the use of radiation in medical settings increases, particularly in nuclear medicine for diagnosis or in treatment as radiotherapy, understanding the effect of radiation on human health and particularly radiation-associated cancer becomes critical. However, studies and conclusive evidence regarding radiation exposure are lacking. In the present study two cohorts, including 278 patients who are all atomic bomb survivors, were examined. Immunohistochemistry was used to determine the expression and localization of fukutin in GC in relation to radiation exposure status.

Although the positive expression of fukutin was not associated with radiation exposure status in the first cohort from HRCHABSH, the second cohort from HUH exhibited a significant association between these two variables. As the HRCHABSH patients were not LSS cohort members, atomic bomb radiation doses may not be correctly estimated. The HRCHABSH patients were classified into a group exposed at a short distance and a group exposed at a long distance according to the distance from the hypocenter of the bombing.
Precise estimation of radiation dose in the LSS cohort required a complicated system with numerous criteria (20). The LSS cohort from the RERF is based on the DS02 system, which was established by the Joint US-Japan Working Group, a collaboration among American, German and Japanese universities together with national laboratories (20). This group revised the gamma ray and neutron fluence calculations of source terms from the previous DS86 system $(20,23,24)$. It may be hypothesized that the difference in estimation strategies for radiation exposure led to the discrepancy between the two cohorts. In addition, as fukutin expression was associated with CD10 expression in the HRCHABSH cohort in the present study, the association between fukutin expression and radiation exposure may be affected by CD10 expression.

The HUH cohort with LSS members (and radiation dose estimation by the DS02 system) demonstrated a statistically significant association between fukutin expression and radiation exposure in the current study. These results suggest that the immunohistochemical analysis of fukutin from surgically resected GC samples is useful to identify radiation-associated GC. Previous studies have demonstrated that high dose-exposed groups exhibit lower levels of versican and osteonectin in tumor-associated stroma (13) and higher expression levels of miR-24 (14), compared with low dose-exposed groups. This suggests that a combination of these markers may help successfully identify radiation-associated GC.

Fukutin is hypothesized to be involved in basement membrane formation. Knockdown of FKTN reduces the binding activity of $\alpha$-dystroglycan for ligands, including laminin, agrin or neurexin (25). This indicates that the loss of fukutin function results in defective glycosylation of $\alpha$-dystroglycan, a key element of the dystrophin-glycoprotein complex, resulting in disruption of the linkage between the 
cytoskeleton and the basal lamina (25). This suggests that alteration of glycosylation may occur in GC in atomic bomb survivors.

In the present study, the expression of fukutin in the LSS cohort demonstrated a significant association with radiation dose exposure; however, the sample size of 86 GC cases may not be sufficient for statistical confirmation. Therefore, further studies with larger sample sizes from the LSS cohort with appropriate radiation dose estimation are required.

In conclusion, several studies have been performed on atomic bomb survivor cohorts for improved understanding of the effects of radiation on human health. The present study detected the expression of fukutin in the low dose-exposed group (LSS cohort), but did not observe an association between fukutin expression levels and radiation exposure status in the HRCHABSH cohort. To clarify whether fukutin may be a potential biomarker to define radiation-induced GC in atomic bomb survivors, future studies in a larger cohort with precise radiation dose estimation are required.

\section{Acknowledgements}

The authors would like to thank Mr. Shinichi Norimura (Hiroshima University, Hiroshima, Japan) for technical assistance and advice. This work was supported by Grants-in-Aid for Scientific Research (grant nos. 25460417 and 15H04713) from the Japan Society for the Promotion of Science.

\section{References}

1. Thompson DE, Mabuchi K, Ron E, Soda M, Tokunaga M, Ochikubo S, Sugimoto S, Ikeda T, Terasaki M and Izumi $S$ Cancer incidence in atomic bomb survivors. Part II: Solid tumors, 1958-1987. Radiat Res 137: (2 Suppl) S17-S67, 1994.

2. Pierce DA, Shimizu Y, Preston DL, Vaeth M and Mabuchi K: Studies of the mortality of atomic bomb survivors. Report 12 , Part I. Cancer: 1950-1990. 1996. Radiat Res 146: 61-87, 2012.

3. Ozasa K, Shimizu Y, Suyama A, Kasagi F, Soda M, Grant EJ, Sakata R, Sugiyama H and Kodama K: Studies of the mortality of atomic bomb survivors, Report 14, 1950-2003: An overview of cancer and noncancer diseases. Radiat Res 177: 229-243, 2012.

4. Sakata R, Grant EJ, Furukawa K, Misumi M, Cullings H, Ozasa K and Shore RE: Long-term effects of the rain exposure shortly after the atomic bombings in Hiroshima and Nagasaki. Radiat Res 182: 599-606, 2014.

5. Global Burden of Disease Cancer Collaboration, Fitzmaurice C, Dicker D, Pain A, Hamavid H, Moradi-Lakeh M, MacIntyre MF, Allen C, Hansen G and Woodbrook R: The global burden of cancer 2013. JAMA Oncol 1: 505-527, 2015.

6. Yasui W, Sentani K, Sakamoto N, Anami K, Naito Y and Oue N: Molecular pathology of gastric cancer: Research and practice. Pathol Res Pract 207: 608-612, 2011

7. Ferlay J, Soerjomataram I, Dikshit R, Eser S, Mathers C, Rebelo M, Parkin DM, Forman D and Bray F: Cancer incidence and mortality worldwide: Sources, methods and major patterns in GLOBOCAN 2012. Int J Cancer 136 E359-E386, 2015

8. Preston DL, Shimizu Y, Pierce DA, Suyama A and Mabuchi K: Studies of mortality of atomic bomb survivors. Report 13: Solid cancer and noncancer disease mortality: 1950-1997. 2003. Radiat Res 178: AV146-AV172, 2012.
9. Preston DL, Ron E, Tokuoka S, Funamoto S, Nishi N, Soda M, Mabuchi K and Kodama K: Solid cancer incidence in atomic bomb survivors: 1958-1998. Radiat Res 168: 1-64, 2007.

10. Takeshima Y, Seyama T, Bennett WP, Akiyama M, Tokuoka S, Inai $\mathrm{K}$, Mabuchi $\mathrm{K}$, Land $\mathrm{CE}$ and Harris CC: p53 mutations in lung cancers from non-smoking atomic-bomb survivors. Lancet 342: 1520-1521, 1993

11. Takahashi K, Eguchi H, Arihiro K, Ito R, Koyama K, Soda M, Cologne J, Hayashi Y, Nakata Y, Nakachi K and Hamatani K: The presence of BRAF point mutation in adult papillary thyroid carcinomas from atomic bomb survivors correlates with radiation dose. Mol Carcinog 46: 242-248, 2007.

12. Iwamoto KS, Mizuno T, Tokuoka S, Mabuchi K and Seyama T: Frequency of p53 mutations in hepatocellular carcinomas from atomic bomb survivors. J Natl Cancer Inst 90: 1167-1168, 1998.

13. Oue N, Sentani K, Sakamoto N, Motoshita J, Nishisaka T, Fukuhara T, Matsuura H, Sasaki H, Nakachi K and Yasui W: Characteristic gene expression in stromal cells of gastric cancers among atomic-bomb survivors. Int J Cancer 124: 1112-1121, 2009.

14. Naito Y, Oue N, Pham TT, Yamamoto M, Fujihara M, Ishida T, Mukai S, Sentani K, Sakamoto N, Hida E, et al: Characteristic miR-24 expression in gastric cancers among atomic bomb survivors. Pathobiology 82: 68-75, 2015

15. Anami K, Oue N, Noguchi T, Sakamoto N, Sentani K, Hayashi T, Hinoi T, Okajima M, Graff JM and Yasui W: Search for transmembrane protein in gastric cancer by the Escherichia coli ampicillin secretion trap: Expression of DSC2 in gastric cancer with intestinal phenotype. J Pathol 221: 275-284, 2010.

16. Oo HZ, Sentani K, Sakamoto N, Anami K, Naito Y, Oshima T, Yanagihara K, Oue N and Yasui W: Identification of novel transmembrane proteins in scirrhous-type gastric cancer by the Escherichia coli ampicillin secretion trap (CAST) method: TM9SF3 participates in tumor invasion and serves as a prognostic factor. Pathobiology 81: 138-148, 2014.

17. Oue N, Sentani K, Sakamoto N and Yasui W: Clinicopathologic and molecular characteristics of gastric cancer showing gastric and intestinal mucin phenotype. Cancer Sci 106: 951-958, 2015.

18. Oo HZ, Sentani K, Mukai S, Hattori T, Shinmei S, Goto K, Sakamoto N, Naito Y, Anami K, Trang PT, et al: Fukutin, identified by the Escherichia coli ampicillin secretion trap (CAST) method, participates in tumor progression in gastric cancer. Gastric Cancer 19: 443-452, 2016

19. Kobayashi K, Nakahori Y, Miyake M, Matsumura K, Kondo-Iida E, Nomura Y, Segawa M, Yoshioka M, Saito K, Osawa M, et al: An ancient retrotransposal insertion causes Fukuyama-type congenital muscular dystrophy. Nature 394: 388-392, 1998.

20. Preston DL, Pierce DA, Shimizu Y, Cullings HM, Fujita S, Funamoto $\mathrm{S}$ and Kodama K: Effect of recent changes in atomic bomb survivor dosimetry on cancer mortality risk estimates. Radiat Res 162: 377-389, 2004.

21. Sobin LH and Compton CC: TNM seventh edition: What's new, what's changed: Communication from the international union against cancer and the American Joint Committee on Cancer. Cancer 116: 5336-5339, 2010

22. Lauren P: The two histological main types of gastric carcinoma: Diffuse and so-called intestinal-type carcinoma. An attempt at a histo-clinical classification. Acta Pathol Microbiol Scand 64: 31-49, 1965.

23. Cullings HM, Fujita S, Funamoto S, Grant EJ, Kerr GD and Preston DL: Dose estimation for atomic bomb survivor studies: Its evolution and present status. Radiat Res 166: 219-254, 2006.

24. Straume T, Rugel G, Marchetti AA, Rühm W, Korschinek G, McAninch JE, Carroll K, Egbert S, Faestermann T, Knie K, et al: Measuring fast neutrons in Hiroshima at distances relevant to atomic-bomb survivors. Nature 424: 539-542, 2003.

25. Saito F, Masaki T, Saito Y, Nakamura A, Takeda S, Shimizu T, Toda T and Matsumura K: Defective peripheral nerve myelination and neuromuscular junction formation in fukutin-deficient chimeric mice. J Neurochem 101: 1712-1722, 2007. 